\title{
An Adaptive Threshold Dynamics Method for Three-Dimensional Wetting on Rough Surfaces
}

\author{
Xianmin $\mathrm{Xu}^{1,2, *}$ and Wenjun Ying $^{3}$ \\ ${ }^{1}$ LSEC, ICMSEC, NCMIS, Academy of Mathematics and Systems Science, Chinese \\ Academy of Sciences, Beijing 100190, China. \\ 2 School of Mathematical Sciences, University of Chinese Academy of Sciences, \\ Beijing 100049, China. \\ 3 School of Mathematical Sciences, MOE-LSC and Institute of Natural Science, \\ Shanghai Jiao Tong University, Shanghai 200240, China.
}

Received 5 November 2019; Accepted (in revised version) 20 March 2020

\begin{abstract}
We propose an adaptive threshold dynamics method for wetting problems in three space dimensions. The method is based on solving a linear heat equation and a thresholding step in each iteration. The heat equation is discretized by a cellcentered finite volume method on an adaptively refined mesh. An efficient technique for volume conservation is developed on the nonuniform meshes based on a quicksorting operation. By this method, we compute some interesting wetting problems on complicated surfaces. Numerical results verify some recent theory for the apparent contact angle on rough and chemically patterned surfaces.
\end{abstract}

AMS subject classifications: 65M08, 65M50, 76T10

Key words: Adaptive, threshold dynamics method, wetting.

\section{Introduction}

Wetting describes how liquid stays and spreads on solid surfaces. It appears in many industrial processes, e.g. painting, printing, oil recovery, etc. One important phenomenon in wetting is that the geometric roughness and chemical inhomogeneity of the solid surface can change dramatically the wetting properties. This is the so-called lotus effect. The property is of critical importance in designing self-cleaning materials and hydrophobic surfaces with low contact angle hysteresis.

Theoretical studies for wetting phenomena on rough and inhomogeneous surfaces are enormous(c.f. $[3,12]$ and the reference therein). The equilibrium wetting problem is

${ }^{*}$ Corresponding author. Email addresses: xmxu@lsec.cc.ac.cn (X. Xu), wying@sjtu.edu.cn (W. Ying) 
to minimize the total interface energy in a solid-liquid-vapor system. When the solid surface is rough, there are usually many local minimizers. By the homogenization method, one can show that the local minimizer corresponds to some modified Wenzel and Cassie equations $[45,49]$. These equations imply that the macroscopic apparent angle is determined by an average of the chemical and geometric properties along the triple contact line $[11,26,33]$. This is different from the classical Wenzel and Cassies equations $[9,43]$, which use a surface average instead of the average on the contact line.

Even though there are many theoretical studies, there still exist many controversies and open questions for wetting on rough surfaces [14,16], especially in three dimensional spaces. The three dimensional wetting problem is much more difficult than that in two dimensions. In the latter case, the contact line is reduced to a point and the apparent contact angle has a simple relation to the local Young's angle at the point $[4,48]$. In three dimensions, the position of the contact line can be very complicated. In general, the location of the contact line is not known a priori and numerical simulations are needed to predict the wetting properties of a rough surface.

Numerical simulation for wetting on rough surfaces is quite challenging, since it is a free interface problem with complex boundary conditions. For example, a phase-field model for wetting includes a nonlinear boundary condition [10,32], which is to enforce the Young's equation for the microscopic contact angle [50]. The boundary condition is difficult to implement numerically. In addition, the possible topological change of the liquid-vapor interface in the Wenzel-Cassie transition on rough surfaces may cause difficulties to other standard numerical methods $[6,13,34,44,51]$.

Recently, a threshold dynamics method is developed for wetting problems in two space dimensions $[41,47]$. The method alternately diffuses and sharpens the characteristic function of a domain with a free boundary. It is easy to implement and quite efficient. The method is motivated by the work of S. Esedoglu and F. Otto [17] and can originate from the well-known MBO method for motion of interfaces driven by the mean curvature(socalled mean curvature flow) [30]. Theoretical studies show that the MBO method has first order convergence when the surface is smooth $[20,22,27,39]$. The threshold dynamics method has also been applied to many other problems $[15,19,31,37,40]$. It is usually implemented on uniform meshes so that a fast Fourier transform (FFT) method can be used. One exception is the work in [23], where a nonuniform FFT is applied.

In this paper, we propose a new adaptive threshold dynamics method for wetting problems on rough surfaces in three space dimensions. To reduce the computational complexity, the mesh is adaptively refined in the neighborhood of the liquid-vapor interface and the rough solid surface while it is coarsened elsewhere. Different from the previous work [47], we do not use the convolution method to diffuse the characteristic functions. We solve a linear heat equation directly by a cell-centered finite volume method on the locally refined grids. The discrete heat equations on the adaptive but locally uniform grids are solved with a fast composite grid iterative method $[7,8,29]$, which further improves efficiency of the algorithm. We also generalize the volume preserving technique in [47] to the case with non-uniform meshes. The technique is based on a quick-sorting operation 
and much more efficient than standard methods.

By the adaptive threshold dynamic method, we compute several wetting problems on rough surfaces which are of interest in real applications. Numerical results show that the apparent contact angle on a chemically patterned surface can be described by a modified Cassie equation and the angle on a geometrically rough surface is characterized by a modified Wenzel equation. This verifies the previous theories for wetting on rough surfaces $[11,26,33,45]$. A Cassie-Baxter state for wetting is observed on a highly rough surface even the droplet is in Wenzel state initially. This shows that our method can easily deal with the topological changes of the liquid-vapor interface. We also do simulations for wetting on curved surfaces and on surfaces with chemical gradient, where there is no equilibrium state in the system. It seems that the method captures the motion of the droplet which driven by a gradient flow of the total surface energies. The numerical results are consistent with the observations in experiments and analysis [28].

Finally, we would like to remark that there are different approaches for discretizing the heat equation and general elliptic partial differential equations on non-uniform grids. Some are node-centered or vertex-centered methods such as the one by Bramble et al. [7]. Some are cell-centered methods such as the one by McCormick and Thomas [29]. We adapt a cell-centered finite volume method in [29] to discretize the heat equation in this work. It assumes that the solution is piecewise constant on each cell. This makes it natural and convenient to compute volumes of cells in a droplet and to sort cells by its volumes in the volume-preserving step.

The structure of the paper is as follows. In Section 2, we introduce the mathematical model of wetting problems. In Section 3, we introduce the adaptive threshold dynamics method, including the theory and the key steps. Some numerical experiments are shown in Section 4, including the wetting problems on rough and chemically patterned surfaces. Finally, some concluding remarks are given in Section 5.

\section{The mathematical model}

\subsection{Minimization of the interface energies}

We consider the wetting problem on a rough solid surface. The shape of a droplet on the solid surface is determined by minimizing the total free energy in the solid-liquidvapor system. When the characteristic length of the droplet is smaller than the capillary length, the gravitational potential energy can be ignored. Then the total energy is a sum of interface energies,

$$
E=\gamma_{L V}\left|\Sigma_{L V}\right|+\gamma_{S V}\left|\Sigma_{S V}\right|+\gamma_{S L}\left|\Sigma_{S L}\right|
$$

where $\Sigma_{L V}, \Sigma_{S V}$ and $\Sigma_{S L}$ denote respectively the liquid-vapor, solid-vapor and solidliquid interfaces, and $\gamma_{L V}, \gamma_{S V}$ and $\gamma_{S L}$ are corresponding interface energy densities. $|\cdot|$ denotes the area of an interface. 

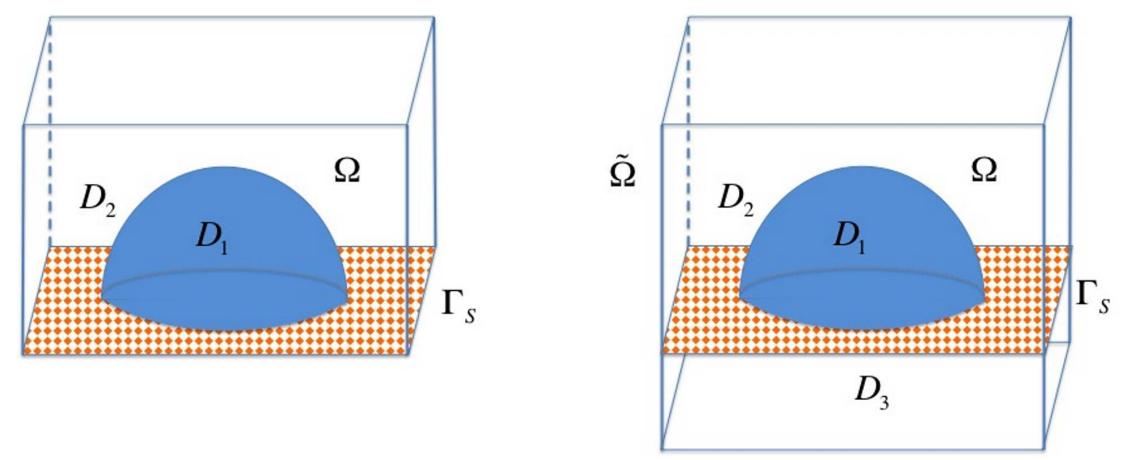

Figure 1: Left: Original domain $\Omega=D_{1} \cup D_{2}$. Right: Extended computational domain $\tilde{\Omega}=\Omega \cup D_{3}$.

Suppose we consider the wetting problem in a domain $\Omega$ (see Fig. 1). Part of its boundary is the solid surface $\Gamma_{S}$. Suppose the domain $\Omega$ is composed of two parts $D_{1}$ and $D_{2}$, representing the liquid region and vapor region. Then we have $\Sigma_{L V}=\partial D_{1} \cap \partial D_{2}$, $\Sigma_{S L}=\partial D_{1} \cap \Gamma_{S}$ and $\Sigma_{S V}=\partial D_{2} \cap \Gamma_{S}$. In equilibrium, the energy is minimized under the constraint that the volume of droplet is constant. Therefore, the mathematical problem is written as

$$
\min _{\left|D_{1}\right|=V_{0}, D_{1} \cup D_{2}=\Omega} E\left(D_{1}, D_{2}\right)=\gamma_{L V}\left|\partial D_{1} \cap \partial D_{2}\right|+\gamma_{S V}\left|\partial D_{2} \cap \Gamma_{S}\right|+\gamma_{S L}\left|\partial D_{1} \cap \Gamma_{S}\right| .
$$

Here $V_{0}>0$ is a given constant number.

The problem is trivial when the solid surface $\Gamma_{S}$ is planar and homogeneous. The minimizer corresponds to a droplet with spherical surface and the contact angle given by the Young's equation [50]

$$
\cos \theta_{Y}=\frac{\gamma_{S V}-\gamma_{S L}}{\gamma_{L V}}
$$

However, it is much more complicated when the solid surface is rough or chemically inhomogeneous. The shape of the droplet is not spheric and the apparent contact angle might not be unique. There are many theoretical studies on how the apparent contact angles depend on the local roughness or chemical inhomogeneity. But there are still some controversies and open problems in this field [16]. Numerical methods for wetting problems on rough surfaces are needed.

\subsection{An approximation model}

Numerical simulations for wetting on rough or chemically patterned surfaces are very difficult. To solve the problem, different numerical methods are developed according to different representations of the free energies. For example, if one approximates the energy in (2.1) by a phase-field function, the energy minimizer can be obtained by solving a Cahn-Hilliard equation with a relaxed boundary condition [10]. However, the boundary condition is nonlinear and still difficult to implement. 
In this paper, we utilize an approximation of the interface energy as used in $[17,47]$. Given $D_{1}, D_{2} \in R^{n}$, with $n=3$ being the dimension of the space, the area of their interface can be approximated by

$$
\left|\partial D_{1} \cap \partial D_{2}\right| \approx \frac{1}{\sqrt{\delta t}} \int \chi_{D_{1}} G_{\delta t} * \chi_{D_{2}} d x
$$

where $\chi_{D_{i}}$ is the characteristic function of $D_{i}$

$$
\chi_{D_{i}}= \begin{cases}1 & \text { if } x \in D_{i} \\ 0 & \text { otherwise; }\end{cases}
$$

and $G_{\delta t}$ is the heat (Gaussian) kernel

$$
G_{\delta t}(\mathbf{x})=\frac{1}{(4 \pi \delta t)^{n / 2}} \exp \left(-\frac{|\mathbf{x}|^{2}}{4 \delta t}\right) \text {. }
$$

Here we assume $\delta t$ is a small number. It is proved that the right-hand side term of (2.3) converges to the left-hand side term when $\delta t$ goes to zero [1]. The approximation can be understood as follows. Notice that $\frac{1}{\sqrt{\delta t}} \int \chi_{D_{1}} G_{\delta t} * \chi_{D_{2}} d x=\frac{1}{\sqrt{\delta t}} \int_{D_{1}} G_{\delta t} * \chi_{D_{2}} d x$. If we consider $G_{\delta t} * \chi_{D_{2}}$ as the solution of a heat equation at $\delta t$ with the initial condition $\chi_{D_{2}}$, the term $\int_{D_{1}} G_{\delta t} * \chi_{D_{2}} d x$ is the total heat flow from $D_{2}$ (with temperature 1) to $D_{1}$ (with temperature 0$)$ in the period $(0, \delta t)$. When $\delta t$ is small, the flow is proportional to the area of their interface(with a coefficient $\sqrt{\delta t}$ ).

In the wetting problem, we also need to approximate the surface energies on the solid boundary. For that purpose, we can extend the domain $\Omega$ to a larger domain $\tilde{\Omega}$, as shown in Figure 1. The domain $D_{3}=\tilde{\Omega} \backslash \Omega$ represents the solid domain. Then we have

$$
\begin{aligned}
& \left|\partial D_{1} \cap \Gamma_{S}\right| \approx \frac{1}{\sqrt{\delta t}} \int \chi_{D_{1}} G_{\delta t} * \chi_{D_{3}} d x ; \\
& \left|\partial D_{2} \cap \Gamma_{S}\right| \approx \frac{1}{\sqrt{\delta t}} \int \chi_{D_{2}} G_{\delta t} * \chi_{D_{3}} d x .
\end{aligned}
$$

Thus the total energy $E\left(D_{1}, D_{2}\right)$ in (2.2) is approximated by

$$
\begin{aligned}
E^{\delta t}\left(\chi_{D_{1}}, \chi_{D_{2}}\right)= & \frac{\gamma_{L V}}{\sqrt{\delta t}} \int \chi_{D_{1}} G_{\delta t} * \chi_{D_{2}} d x+\frac{\gamma_{S L}}{\sqrt{\delta t}} \int \chi_{D_{1}} G_{\delta t} * \chi_{D_{3}} d x \\
& +\frac{\gamma_{S V}}{\sqrt{\delta t}} \int \chi_{D_{2}} G_{\delta t} * \chi_{D_{3}} d x .
\end{aligned}
$$

We introduce a functional space for the characteristic functions that

$$
\mathcal{B}=\left\{\left(u_{1}, u_{2}\right) \mid u_{i} \in B V(\Omega), u_{i}(x)=0 \text { or } 1, u_{1}(x)+u_{2}(x)=1, \int_{\Omega} u_{1} d x=V_{0}\right\} .
$$

Using this approximation, the problem (2.2) is reduced to

$$
\min _{\left(\chi_{D_{1}}, \chi_{D_{2}}\right) \in \mathcal{B}} E^{\delta t}\left(\chi_{D_{1}}, \chi_{D_{2}}\right) \text {. }
$$

In the following, we develop an efficient numerical method for the problem (2.6). 


\section{Numerical methods}

\subsection{Derivation of the threshold dynamics scheme}

We will propose an iterative method to minimize the modified energy in (2.6). For a given liquid domain $D_{1}^{(k)}$ and a vapor domain $D_{2}^{(k)}=\Omega \backslash D_{1}^{(k)}$, we will find two new domains $D_{1}^{(k+1)}$ and $D_{2}^{(k+1)}$ so that the energy decays

$$
E^{\delta t}\left(\chi_{D_{1}^{(k+1)},} \chi_{D_{2}^{(k+1)}}\right)<E^{\delta t}\left(\chi_{D_{1}^{(k)}}, \chi_{D_{2}^{(k)}}\right) .
$$

For that purpose, we will first linearize $E^{\delta t}\left(\chi_{D_{1}}, \chi_{D_{2}}\right)$ near $\left(\chi_{D_{1}^{(k)}}, \chi_{D_{2}^{(k)}}\right)$ that

$$
E^{\delta t}\left(\chi_{D_{1}}, \chi_{D_{2}}\right)=E^{\delta t}\left(\chi_{D_{1}^{(k)}}, \chi_{D_{2}^{(k)}}\right)+\mathcal{L}\left(\chi_{D_{1}}-\chi_{D_{1}^{(k)}}, \chi_{D_{2}}-\chi_{D_{2}^{(k)}}, \chi_{D_{1}^{(k)}}, \chi_{D_{2}^{(k)}}\right)+\text { h.o.t., }
$$

where

$$
\begin{aligned}
& \mathcal{L}\left(\chi_{D_{1}}-\chi_{D_{1}^{(k)}}, \chi_{D_{2}}-\chi_{D_{2}^{(k)}}, \chi_{D_{1}^{(k)}}, \chi_{D_{2}^{(k)}}\right) \\
= & \mathcal{L}\left(\chi_{D_{1}}, \chi_{D_{2}}, \chi_{D_{1}^{(k)}}, \chi_{D_{2}^{(k)}}\right)-\mathcal{L}\left(\chi_{D_{1}^{(k)},} \chi_{D_{2}^{(k)}}, \chi_{D_{1}^{(k)}}, \chi_{D_{2}^{(k)}}\right)
\end{aligned}
$$

and

$$
\begin{aligned}
& \mathcal{L}\left(\chi_{D_{1}}, \chi_{D_{2}}, D_{1}^{(k)}, D_{2}^{(k)}\right) \\
= & \frac{1}{\sqrt{\delta t}}\left(\int \chi_{D_{1}} G_{\delta t} *\left(\gamma_{L V} \chi_{D_{2}^{(k)}}+\gamma_{S L} \chi_{D_{3}}\right) d x+\int \chi_{D_{2}} G_{\delta t} *\left(\gamma_{L V} \chi_{D_{1}^{(k)}}+\gamma_{S V} \chi_{D_{3}}\right) d x\right) .
\end{aligned}
$$

We first minimize the linear functional $\mathcal{L}\left(\chi_{D_{1}}, \chi_{D_{2}}, \chi_{D_{1}^{(k)}}, \chi_{D_{2}^{(k)}}\right)$ in $\mathcal{B}$. The following lemma shows that the minimizer can be obtained by a simple thresholding approach.

Lemma 3.1. Denote

$$
\phi(x)=\frac{1}{\sqrt{\delta t}} G_{\delta t} *\left[\gamma_{L V}\left(\chi_{D_{2}^{(k)}}-\chi_{D_{1}^{(k)}}\right)+\left(\gamma_{S L}-\gamma_{S V}\right) \chi_{D_{3}}\right] .
$$

Let

$$
D_{1}^{(k+1)}=\{x \in \Omega \mid \phi(x)<\delta\}
$$

for some $\delta$ such that $\left|D_{1}^{(k+1)}\right|=V_{0}$. Define $D_{2}^{(k+1)}=\Omega \backslash D_{1}^{(k+1)}$. Then $\left(\chi_{D_{1}^{(k+1)}, \chi_{D_{2}^{(k+1)}}}\right)$ minimizes $\mathcal{L}\left(\chi_{D_{1}}, \chi_{D_{2}}, \chi_{D_{1}^{(k)}}, \chi_{D_{2}^{(k)}}\right)$ in $\mathcal{B}$.

Proof. By definition, we have $\chi_{D_{1}}+\chi_{D_{2}}=\chi_{\Omega}$. Then the linear functional $\mathcal{L}$ can be rewritten as

$$
\begin{aligned}
\mathcal{L}\left(\chi_{D_{1}}, \chi_{D_{2}}, \chi_{D_{1}^{(k)}}, \chi_{D_{2}^{(k)}}\right)= & \frac{1}{\sqrt{\delta t}} \int \chi_{D_{1}} G_{\delta t} *\left[\gamma_{L V}\left(\chi_{D_{2}^{(k)}}-\chi_{D_{1}^{(k)}}\right)+\left(\gamma_{S L}-\gamma_{S V}\right) \chi_{D_{3}}\right] d x \\
& +\frac{1}{\sqrt{\delta t}} \int \chi_{\Omega} G_{\delta t} *\left(\gamma_{L V} \chi_{D_{1}^{(k)}}+\gamma_{S V} \chi_{D_{3}}\right) d x \\
= & I_{1}+I_{2} .
\end{aligned}
$$


Since $I_{2}$ is a constant independent of $\left(\chi_{D_{1}}, \chi_{D_{2}}\right)$, we need only to minimize $I_{1}$. Notice that

$$
I_{1}=\frac{1}{\sqrt{\delta t}} \int_{D_{1}} G_{\delta t} *\left[\gamma_{L V}\left(\chi_{D_{2}^{(k)}}-\chi_{D_{1}^{(k)}}\right)+\left(\gamma_{S L}-\gamma_{S V}\right) \chi_{D_{3}}\right] d x=\int_{D_{1}} \phi(x) d x .
$$

Clearly, the minimizer of this integral with respect to $D_{1} \subset \Omega$ such that $\left|D_{1}\right|=V_{0}$ is given by the set defined in (3.5). This ends the proof of the lemma.

More importantly we can prove the following theorem. It is the basis of our numerical method.

Theorem 3.1. Suppose $\left(\chi_{D_{1}^{(k+1)},} \chi_{D_{2}^{(k+1)}}\right) \in \mathcal{B}$ is obtained by the process defined in Lemma 3.1. We have

$$
E^{\delta t}\left(\chi_{D_{1}^{(k+1)}}, \chi_{D_{2}^{(k+1)}}\right) \leq E^{\delta t}\left(\chi_{D_{1}^{(k)}}, \chi_{D_{2}^{(k)}}\right),
$$

for all $\delta t>0$. Furthermore, the equality holds only when $D_{1}^{(k+1)}=D_{1}^{(k)}$ and $D_{2}^{(k+1)}=D_{2}^{(k)}$.

Proof. We first rewrite $E^{\delta t}\left(\chi_{D_{1}^{(k+1)},} \chi_{D_{2}^{(k+1)}}\right)$ as:

$$
\begin{aligned}
E^{\delta t}\left(\chi_{D_{1}^{(k+1)}}, \chi_{D_{2}^{(k+1)}}\right)= & E^{\delta t}\left(\chi_{D_{1}^{(k)}}, \chi_{D_{2}^{(k)}}\right)+\mathcal{L}\left(\chi_{D_{1}^{(k+1)},} \chi_{D_{2}^{(k+1)},} \chi_{D_{1}^{(k)}}, \chi_{D_{2}^{(k)}}\right) \\
& -\mathcal{L}\left(\chi_{D_{1}^{(k)},} \chi_{D_{2}^{(k)},} \chi_{D_{1}^{(k)}}, \chi_{D_{2}^{(k)}}\right) \\
& -\frac{\gamma_{L V}}{\sqrt{\delta t}} \int\left(\chi_{D_{1}^{(k+1)}}-\chi_{D_{1}^{(k)}}\right) G_{\delta t} *\left(\chi_{D_{1}^{(k+1)}}-\chi_{D_{1}^{(k)}}\right) d x
\end{aligned}
$$

By the above lemma, we know

$$
\mathcal{L}\left(\chi_{D_{1}^{(k+1)},} \chi_{D_{2}^{(k+1)},}, \chi_{D_{1}^{(k)}}, \chi_{D_{2}^{(k)}}\right) \leq \mathcal{L}\left(\chi_{D_{1}^{(k)}}, \chi_{D_{2}^{(k)}}, \chi_{D_{1}^{(k)}}, \chi_{D_{2}^{(k)}}\right) .
$$

Therefore, we have

$$
\begin{aligned}
E^{\delta t}\left(\chi_{D_{1}^{(k+1)},} \chi_{D_{2}^{(k+1)}}\right) \leq & E^{\delta t}\left(\chi_{D_{1}^{(k)},} \chi_{D_{2}^{(k)}}\right) \\
& -\frac{1}{\sqrt{\delta t}} \int\left(\chi_{D_{1}^{(k+1)}}-\chi_{D_{1}^{(k)}}\right) G_{\delta t} *\left(\chi_{D_{1}^{(k+1)}}-\chi_{D_{1}^{(k)}}\right) d x \\
\leq & E^{\delta t}\left(\chi_{D_{1}^{(k)},} \chi_{D_{2}^{(k)}}\right) .
\end{aligned}
$$

In the last equation, the equality holds only when $D_{1}^{(k+1)}=D_{1}^{(k)}$. This ends the proof of the theorem.

By the above analysis, for give characteristic functions $\left(\chi_{D_{1}^{(k)}}, \chi_{D_{2}^{(k)}}\right) \in \mathcal{B}$, we find a simple way to find new functions $\left(\chi_{D_{1}^{(k+1)},} \chi_{D_{2}^{(k+1)}}\right) \in \mathcal{B}$ such that

$$
E^{\delta t}\left(\chi_{D_{1}^{(k+1)}}, \chi_{D_{2}^{(k+1)}}\right) \leq E^{\delta t}\left(\chi_{D_{1}^{(k)},} \chi_{D_{2}^{(k)}}\right) .
$$

They are given by two steps: 
- (Convolution) Compute $\phi(x)=\frac{1}{\sqrt{\delta t}} G_{\delta t} *\left[\gamma_{L V}\left(\chi_{D_{2}^{(k)}}-\chi_{D_{1}^{(k)}}\right)+\left(\gamma_{S L}-\gamma_{S V}\right) \chi_{D_{3}}\right]$.

- (Thresholding) Find a $\delta>0$ to make sure the domain $D_{1}^{(k+1)}=\{x \in \Omega \mid \phi(x)<\delta\}$ has a volume $V_{0}$ (i.e. $\left|D_{1}^{(k+1)}\right|=V_{0}$ ). Then we set $D_{2}^{(k+1)}=\Omega \backslash D_{1}^{(k+1)}$.

\subsection{Numerical solution of the heat equation}

One key step in the process is to compute the convolution $\phi$ for given domains $D_{1}^{(k)}$ and $D_{2}^{(k)}$. They represent respectively the liquid and vapor domains. It is known that the convolution of a function and a Gaussian kernel is a solution of a heat equation in $R^{3}$ with the initial condition given by the function. The function $\phi$ can be computed as follows. First solve the equation under some proper boundary condition,

$$
\begin{cases}\partial_{t} u-\Delta u=0, & 0<t<\delta t \\ u(x, 0)=\gamma_{L V}\left(\chi_{D_{2}}-\chi_{D_{1}}\right)+\left(\gamma_{S L}-\gamma_{S V}\right) \chi_{D_{3},} & t=0\end{cases}
$$

and then set $\phi(x)=u(x, \delta t)$ after scaling. Theoretically, we need to propose a periodic boundary condition for the equation. For that purpose, we have to extend $\Omega$ to a larger domain symmetrically. This will increase the degree of freedoms by times. For simplicity, we assume the liquid domain $D_{1}$ is always inside $\Omega$ and far from the boundary of $\Omega$. Then we can use a Dirichlet or Neumann boundary condition on $\partial \tilde{\Omega}$. When $\delta t$ is small, the effect of the boundary condition on the threshold dynamics method can be ignored. In our simulations, the boundary values of $u(x, t)$ on $\partial \tilde{\Omega}$ are fixed in the time interval $(0, \delta t)$, i.e., all the same as the initial $u(x, 0)$.

If the domain $\tilde{\Omega}$ is partitioned uniformly, the standard FFT method can also be used to solve the heat equation. However, the uniform mesh may be very inefficient when the solid surface $\Gamma$ is rough. Suppose the roughness can be characterized by a small parameter $\epsilon \ll 1$. In that case, a very fine mesh with mesh size $h<\epsilon$ is needed to resolve the local geometry properties. Then the total degree of freedoms is scaled as $\mathcal{O}\left(h^{-3}\right)$, which is much larger than $\mathcal{O}\left(\epsilon^{-3}\right)$. To improve the efficiency, a natural idea is to use adaptively refined meshes. In this case, the resulting problem will have a degree of freedoms of order $\mathcal{O}\left(\epsilon^{-2}\right)$. The adaptive method decreases largely the computational complexity when $\epsilon$ is small.

The problem (3.7) can be solved by many standard numerical methods. In our simulations, time integration of the heat equation is done by the backward Euler method to avoid time step restriction; space discretization and the resulting discrete equations are handled with adaptive mesh refinement and multilevel composite grid iteration techniques $[7,8,29]$.

The adaptive multilevel method is briefly described as follows. At the very beginning of the numerical solution, the domain $\tilde{\Omega}$ is discretized into a uniform Cartesian mesh with mesh size $h_{0} \gg \delta t$. In each time step, including the first one, the region near the boundary 

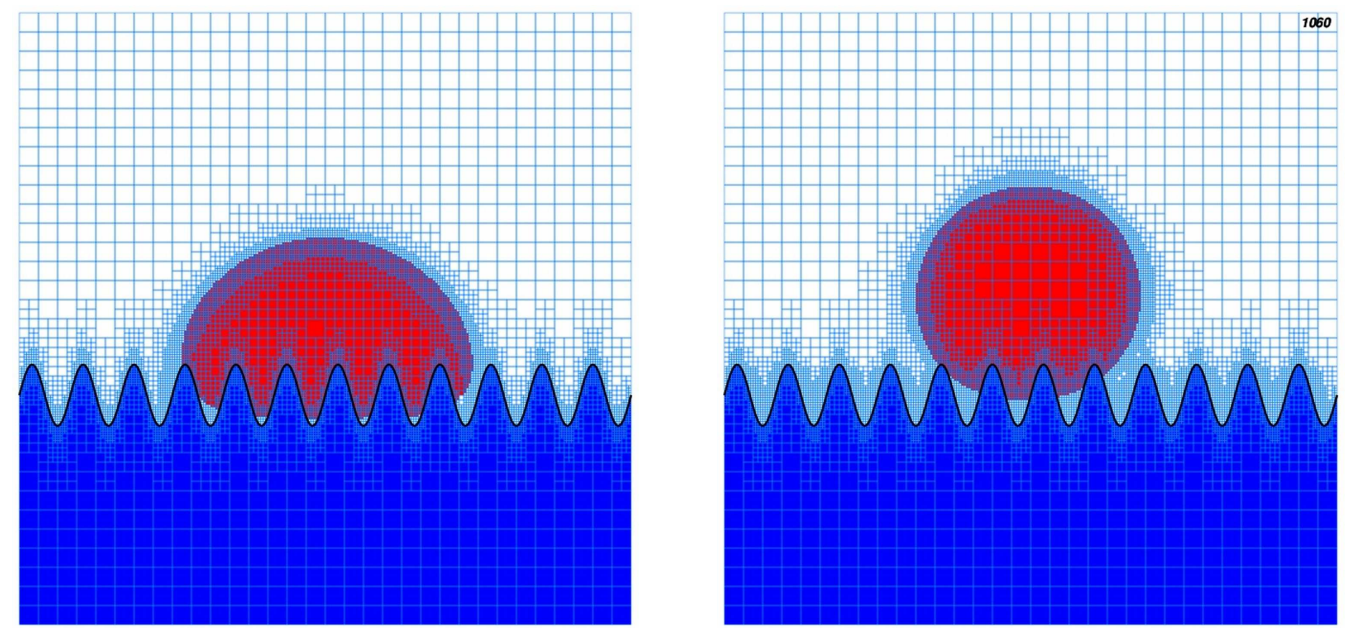

Figure 2: The adaptive mesh for droplet with different shape in two space dimensions.

of $D_{1}$ is locally refined so that a sequence of locally refined grids are generated (see Fig. 2 for a composite grid generated by the method in two dimensions). In the adaptive mesh refinement, the refinement factor between two consecutive grids is fixed to be two and a rule is imposed to guarantee that the ratio of edge lengths between any two adjacent cells/sides on the resulting composite grid is not greater than two.

Space discretization of the heat equation on the locally refined composite grid is done with a cell-centered finite volume method, which is different from but similar to the one by McCormick [29]. Associated with the center of a regular cell, whose neighboring cells all have the same size, the finite volume stencil for the Laplacian operator takes the same form as the standard one on a uniform grid. Associated with the center of an irregular cell, some of whose neighboring cells may be larger or smaller than itself, the finite volume stencil is modified. The finite volume stencil there is constructed by weak continuity of the solution and conservation of the numerical flux across the coarsefine grid interface. Fig. 3 shows finite volume stencils, ignoring the $h^{-2}$ factors, for the negative Laplacian operator on a composite grid in two space dimensions by the method. The values at cell centers in Fig. 3 are diagonal entries of the coefficient matrix of the finite volume system. The values at cell edges in Fig. 3 are the off-diagonal coefficients in the finite volume stencils associated with the corresponding cell centers. Details of the finite volume discretization of the heat equation and the corresponding multilevel/multigrid iteration for the discrete equations on the locally refined grids in two and three space dimensions are omitted here and will be reported separately somewhere else.

\subsection{Volume conservation}

The other important step of the process in Section 3.1 is to preserve the volume of the fluid domain. This is to find a $\delta$ to make sure $\left|D_{1}^{(k+1)}\right|=V_{0}$. Usually, it is not an easy 


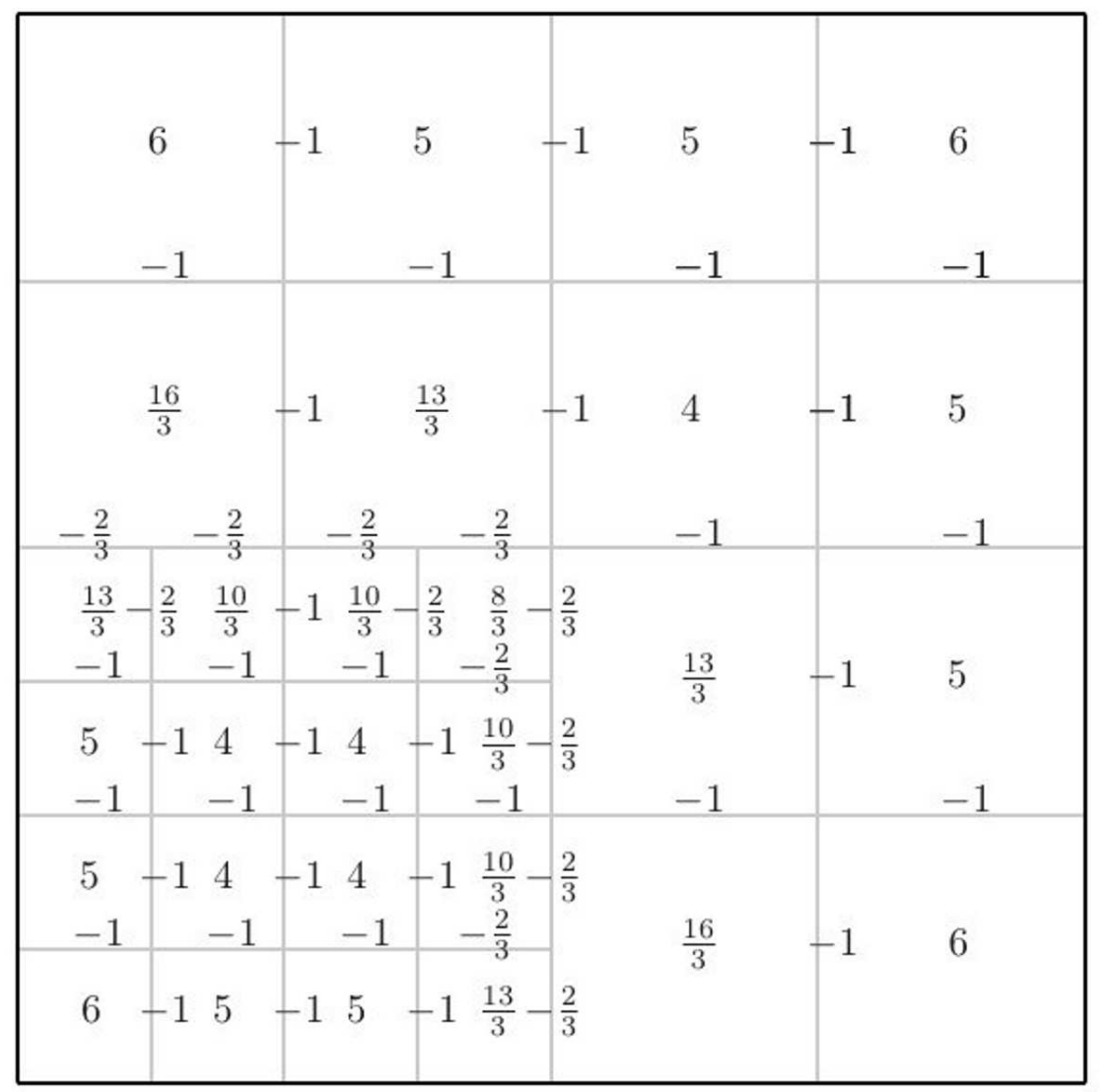

Figure 3: Cell-centered finite volume stencils (omitting the $h^{-2}$ factors) for the negative Laplacian operator(with Dirichlet boundary condition) on a composite grid in two space dimensions.

problem to compute $\delta$ and the standard method is by the Newton method $[25,36,38]$. In [47], a technique based on fast sorting scheme is developed to find $\delta$ efficiently in the case of uniform meshes. In two dimensions, let $\left\{\phi_{i j}\right\}$ be the value of $\phi$ on the grid points. If we sort $\phi_{i j}$ in monotonely increasing order and denote the sequence as $\Phi$, then one can simply let $\delta=(\Phi[M]+\Phi[M+1]) / 2$, where $M=\left[V_{0} / h_{1}^{2}\right]$ is the integer part of $V_{0} / h_{1}^{2}$ and $h_{1}$ is the mesh size of the uniform mesh. We will generate this method to three dimensional case with non-uniform meshes.

For simplicity, we denote by $\phi_{i}$ the value of $\phi$ at the $i^{\text {th }}$ cell. Volume of the $i^{\text {th }}$ cell is denoted as $a_{i}$. We sort $\phi_{i}$ in monotonely increasing order into a sequence $\Phi$. The order of $a_{i}$ will be changed accordingly. We denote the sequence as $A$. We compute the sum of volumes of the first $k$ cells in the sequence $A$ and denote it as

$$
S_{k}=\sum_{i=1}^{k} A[i]
$$


We choose $M$ to be the integer so that

$$
S_{M}<V_{0}<S_{M+1}
$$

and set $\delta$ as

$$
\delta=(\Phi[M]+\Phi[M+1]) / 2 .
$$

This is used in the thresholding step to define $D_{1}^{(k+1)}=\{x \in \Omega: \phi<\delta\}$.

We assume that the adaptive mesh is almost uniform locally in a narrow band near the liquid-vapor interface. The local mesh size is $h$. Then the volume of each cell in the narrow band is given by $h^{3}$. It is easy to see that the numerical errors for the volume $\left|D_{1}^{(k+1)}\right|$ generated by the above method is of order $\mathcal{O}\left(h^{3}\right)$.

\subsection{The algorithms}

Combining the above analysis, we obtain the following threshold dynamics method for wetting problems on solid surfaces.

\section{Algorithm 1:}

Step 0. Given initial $D_{1}^{0}, D_{2}^{0} \subset \Omega$, such that $D_{1}^{0} \cap D_{2}^{0}=\varnothing, D_{1}^{0} \cup D_{2}^{0}=\Omega$ and $\left|D_{1}^{0}\right|=V_{0}$. Set a tolerance parameter $\varepsilon>0$, the smallest mesh size $h$ and $\delta t=\mathcal{O}(h)$.

Step 1. Solve the heat equation

$$
\begin{cases}\partial_{t} u-\Delta u=0, & 0<t<\delta t, \\ u(x, 0)=\gamma_{L V}\left(\chi_{D_{2}^{k}}-\chi_{D_{1}^{k}}\right)+\left(\gamma_{S L}-\gamma_{S V}\right) \chi_{D_{3},} & t=0,\end{cases}
$$

on an adaptively refined triangulation $\mathcal{T}_{h}$ and set $\phi(x)=u(x, \delta t)$.

Step 2. Find a $\delta$ by the technique in Section 3.3 so that the set

$$
\tilde{D}_{1}^{\delta}=\{x \in \Omega \mid \phi<\delta .\}
$$

satisfies $\left|\tilde{D}_{1}^{\delta}\right|=V_{0}$. Denote $D_{1}^{k+1}=\tilde{D}_{1}^{\delta}$ and $D_{2}^{k+1}=\Omega \backslash D_{1}^{k+1}$.

Step 3. If $\left|D_{1}^{k}-D_{1}^{k+1}\right| \leq \varepsilon$, stop. Otherwise, go back to Step 1 .

One key parameter in the threshold dynamics method is the time step size $\delta t$. It can not be too small. Otherwise, motion of the level-set of the solution of the heat equation in one iteration is too little so that the threshold step will make the domain of the liquid phase unchanged and the method will fail. The standard choice of time step satisfies $\delta t \geq c_{0} \delta x$ [30]. For a mean-curvature flow problem, when the free interface is smooth, the accuracy of the method is of order $\mathcal{O}(\delta t)$. However, when there is a triple junction, convergence rate of the threshold dynamics method is of order $\mathcal{O}\left(\delta t^{1 / 2}\right)[35,47]$. To improve 
the accuracy, we can use the time-adaptive technique [47]. Then we obtain an algorithm as illustrated below. The main steps are the same as the previous algorithm. One key difference is that we add a step 4 to adjust the time step $\delta t$.

\section{Algorithm 2:}

Step 0. Given initial $D_{1}^{0}, D_{2}^{0} \subset \Omega$, such that $D_{1}^{0} \cap D_{2}^{0}=\varnothing, D_{1}^{0} \cup D_{2}^{0}=\Omega$ and $\left|D_{1}^{0}\right|=V_{0}$. Set a tolerance parameter $\varepsilon>0$, a mesh size $h>0, \delta t=\mathcal{O}(h)$ and a constant $c_{1}$.

Step 1. Solve the heat equation

$$
\begin{cases}\partial_{t} u-\Delta u=0, & 0<t<\delta t, \\ u(x, 0)=\gamma_{L V}\left(\chi_{D_{2}^{k}}-\chi_{D_{1}^{k}}\right)+\left(\gamma_{S L}-\gamma_{S V}\right) \chi_{D_{3},} & t=0,\end{cases}
$$

on an adaptively refined triangulation $\mathcal{T}_{h}$ and set $\phi(x)=u(x, \delta t)$.

Step 2. Find a $\delta$ by the technique in Section 3.3 so that the set

$$
\tilde{D}_{1}^{\delta}=\{x \in \Omega \mid \phi<\delta .\}
$$

satisfies $\left|\tilde{D}_{1}^{\delta}\right|=V_{0}$. Denote $D_{1}^{k+1}=\tilde{D}_{1}^{\delta}$ and $D_{2}^{k+1}=\Omega \backslash D_{1}^{k+1}$.

Step 3. If $\left|D_{1}^{k}-D_{1}^{k+1}\right| \leq \varepsilon$, go to Step 4. Otherwise, go back to Step 1 .

Step 4. If $\delta t<c_{1} h^{2}$, stop. Otherwise, set $\delta t=\delta t / 2$. Go back to Step 1 .

In our simulations, we simply set $c_{1}=1$. The optimal accuracy of Algorithm 2 is of the first order with respect to the spacial mesh size $h$. This is due to a discrete characteristic function to approximate the liquid domain. To further improve the accuracy of the method, one can use a signed distance function as in [18]. In addition, to improve the local accuracy near the contact line, the technique of using different scaled parameters in the approximation of the interface energies can also be used [41].

\section{Numerical experiments}

\subsection{Accuracy check of the algorithms}

We first test the numerical method by considering a simple wetting problem on a planar surface. In this case, the equilibrium state of a droplet is known explicitly. The liquid droplet has a spherical surface and a contact angle equal to the Young's angle.

In this example, we set $\Omega=(-1,1) \times(-1,1) \times(0,1.25), \Gamma_{S}=\left\{x \mid x_{3}=0\right\}$. We can set $\tilde{\Omega}=(-1,1) \times(-1,1) \times(-0.75,1.25)$. Let $\Gamma_{L V}=1$ and $\Gamma_{S V}-\Gamma_{S L}=\cos \theta_{Y}$ with $\theta_{Y}=120^{\circ}$. Initially, $D_{1}^{0}=\{x|| x \mid<0.3\}$ which is the hemisphere.

Fig. 4 shows the initial shape and final shape of the droplet. It is clear to see that the equilibrium state is correctly obtained. Furthermore, we list the errors in Table 1 computed by Algorithm 1 . Here the mesh size denotes the smallest grid size in the adaptive 

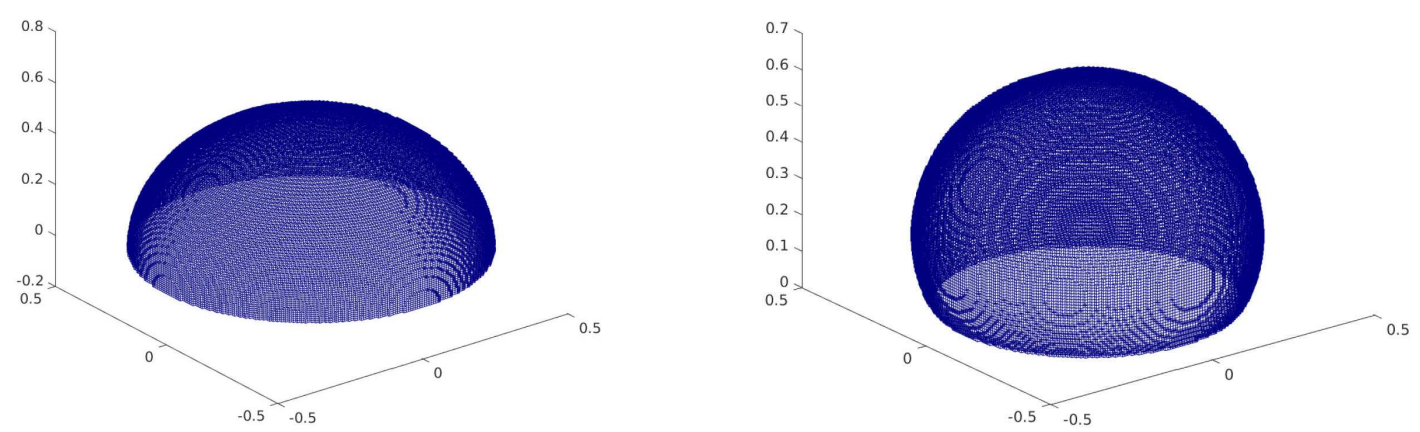

Figure 4: Left: The initial shape of a droplet. Right: The final shape of the $\operatorname{droplet}\left(\theta_{Y}=\frac{2 \pi}{3}\right)$.

Table 1: Accuracy check for computational errors by Algorithm 1.

\begin{tabular}{||c|c|c|c|c||}
\hline$\delta t=h$ & $L^{\infty}$ error & $\begin{array}{c}\text { Convergence } \\
\text { rate }\end{array}$ & $L^{2}$ error & $\begin{array}{c}\text { Convergence } \\
\text { rate }\end{array}$ \\
\hline $1 / 16$ & 0.2394 & - & 0.2229 & - \\
\hline $1 / 32$ & 0.1169 & 1.03 & 0.1102 & 1.02 \\
\hline $1 / 64$ & 0.0625 & 0.90 & 0.0577 & 0.93 \\
\hline $1 / 128$ & 0.0332 & 0.91 & 0.0324 & 0.83 \\
\hline $1 / 256$ & 0.0218 & 0.61 & 0.0211 & 0.62 \\
\hline
\end{tabular}

Table 2: Accuracy check for computational errors by Algorithm 2.

\begin{tabular}{||c|c|c|c|c||}
\hline$h$ & $L^{\infty}$ error & $\begin{array}{c}\text { Convergence } \\
\text { rate }\end{array}$ & $L^{2}$ error & $\begin{array}{c}\text { Convergence } \\
\text { rate }\end{array}$ \\
\hline $1 / 16$ & 0.1572 & - & 0.1258 & - \\
\hline $1 / 32$ & 0.0778 & 1.01 & 0.0632 & 0.99 \\
\hline $1 / 64$ & 0.0411 & 0.92 & 0.0363 & 0.80 \\
\hline $1 / 128$ & 0.0217 & 0.92 & 0.0204 & 0.83 \\
\hline $1 / 256$ & 0.0118 & 0.89 & 0.0113 & 0.85 \\
\hline
\end{tabular}

triangulation. Here we set $\delta t=h$. We could see that the convergence rate is slightly better than the optimal convergence order $\mathcal{O}\left(\delta t^{1 / 2}\right)$. The convergence rate can be further improved if a time-adaptive method (Algorithm 2 ) is used. The results are given in Table 2. The convergence rate is almost of order $\mathcal{O}(h)$. That is because we use the time adaptive technique in Algorithm 2 and the stop criteria is $\delta t \leq c_{1} h^{2}$.

Notice that the accuracy of the threshold dynamics methods depends mainly on the mesh size near the surface of a droplet. Therefore, the adaptive method has almost the 
Table 3: Number of freedoms (NoF).

\begin{tabular}{||c|c|c|c|c||}
\hline$h$ & $\begin{array}{c}\text { Adaptive NoF } \\
\left(N_{a d p}\right)\end{array}$ & $\begin{array}{c}\text { Rate } \alpha \\
\left(N_{a d p} \propto h^{-\alpha}\right)\end{array}$ & $\begin{array}{c}\text { Uniform NoF } \\
\left(N_{\text {uni }}\right)\end{array}$ & $\begin{array}{c}\text { Ratio } \\
\left(N_{\text {uni }} / N_{\text {adp }}\right)\end{array}$ \\
\hline $1 / 16$ & $1.80 \times 10^{4}$ & - & $3.27 \times 10^{4}$ & 1.82 \\
\hline $1 / 32$ & $7.64 \times 10^{4}$ & 2.08 & $2.62 \times 10^{5}$ & 3.43 \\
\hline $1 / 64$ & $3.13 \times 10^{5}$ & 2.04 & $2.10 \times 10^{6}$ & 6.69 \\
\hline $1 / 128$ & $1.28 \times 10^{6}$ & 2.03 & $1.68 \times 10^{7}$ & 13.13 \\
\hline $1 / 256$ & $5.17 \times 10^{6}$ & 2.02 & $1.34 \times 10^{8}$ & 25.96 \\
\hline
\end{tabular}

same accuracy with the standard threshold dynamics method using uniform meshes [47] when their mesh sizes are the same near the surface. However, the computational costs of the adaptive method can be much smaller than the standard method. To see this clearly, we illustrate the total number of freedoms for the two methods in Table 3. Notice that the total number of freedoms $N_{a d p}$ in the adaptive method can changes when the shape of a droplet evolves in one simulation. We show the largest number for $N_{a d p}$ in each case in Table 3. It is easy to see that $N_{a d p}$ increases in an order of $\mathcal{O}\left(h^{-2}\right)$ with respect to the decreasing mesh size $h$. That is because we refine the mesh mainly in the vicinity of the surface of the droplet. In comparison, the total number of freedoms $N_{u n i}$ for the method on uniform meshes increases in an order of $\mathcal{O}\left(h^{-3}\right)$ for a three dimensional problem. When $h$ becomes smaller, the adaptive method has much less freedoms than the standard threshold dynamics method. In addition, although the adaptive method needs some extra cost to refine and coarse the meshes during simulations, the cost is relatively smaller than other operations in the algorithm. Overall our method is more efficient than the standard method on uniform meshes.

In the end of this subsection, we show a numerical example for wetting with topology changes, similar simulations have been done in [42] in two dimensions. As shown in Fig. 5, two spherical droplets with radius $r=0.2$ are set on a planar substrate with Young's angle $\theta_{Y}=\frac{\pi}{3}$. The center of the two droplets are respectively $(0.25,0,0)$ and $(-0.25,0,0)$ and the initial contact angles are equal to $\frac{\pi}{2}$. Since the Young's angle of the substrate is smaller than $\frac{\pi}{2}$, the two droplets will spread outward to minimize the total surface energy. Then they merge into a bigger droplet and its shape evolves gradually to spherical. In the final state, the contact angle is almost equal to the Young's angle $\frac{\pi}{3}$. This example shows that the threshold dynamics method can easily dual with topology changes.

\subsection{Wetting on chemically patterned surfaces}

We then consider a wetting problem on a chemically inhomogeneous surface. For simplicity, suppose the solid is composed of two materials with different contact angles $\theta_{Y 1}$ and $\theta_{Y 2}$. The distribution of two materials in the flat surface is described by a function 

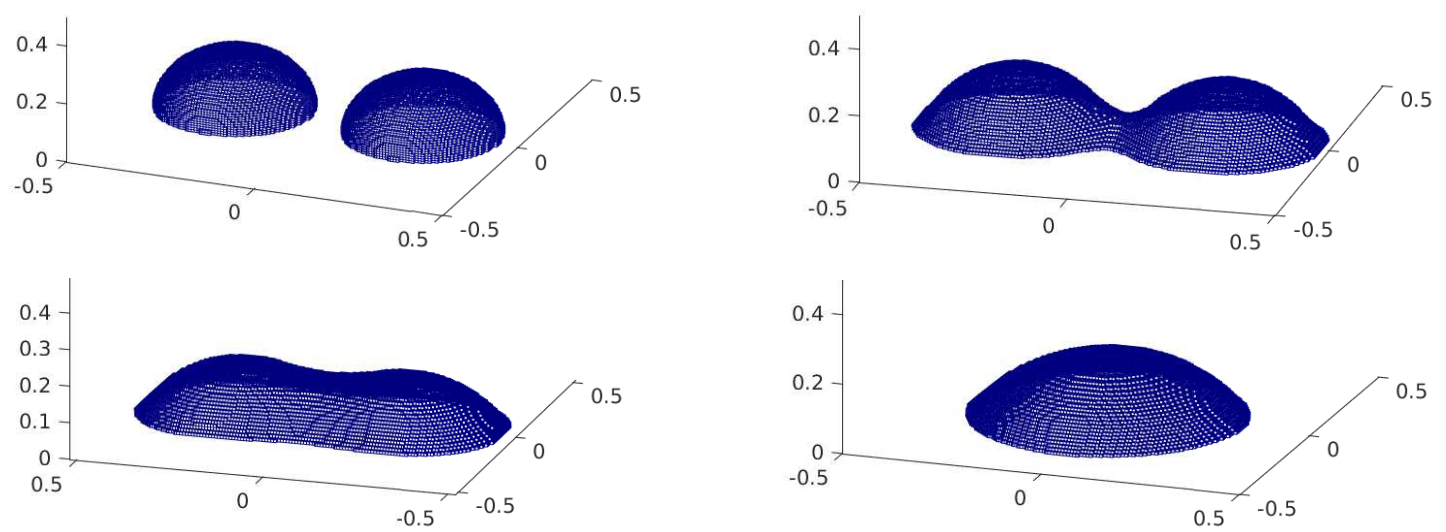

Figure 5: Two droplets merge on a planar surface with Young's angle $\theta_{Y}=\frac{\pi}{3}$.
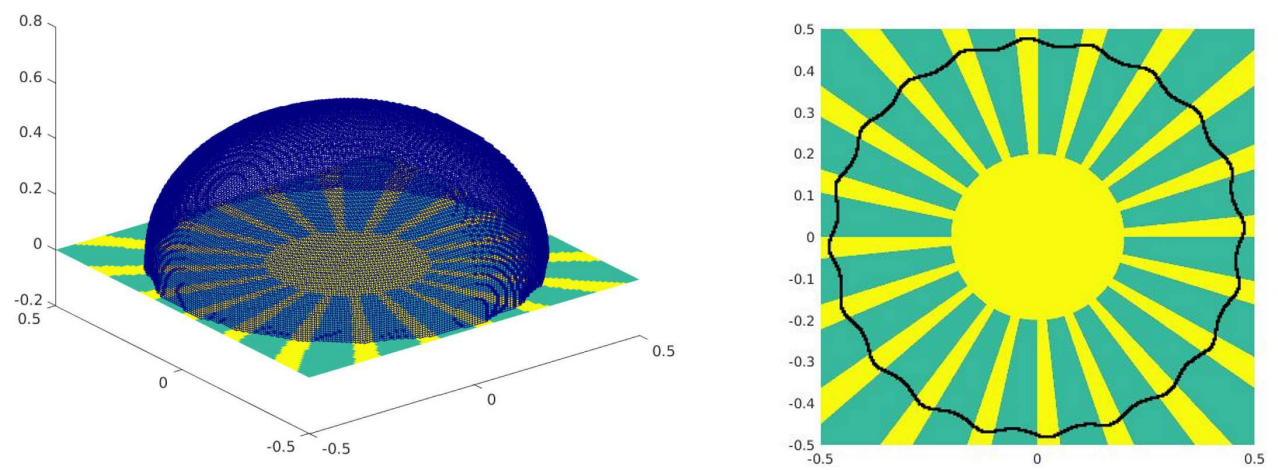

Figure 6: The shape of a droplet on the chemically patterned surface with Young's angle $\theta_{Y 1}=60^{\circ}$ and $\theta_{Y 2}=90^{\circ}$ (left) and the corresponding contact line on the patterned surface (right).

$\theta_{Y}\left(x_{1}, x_{2}\right)$ which is defined as follows,

$$
\theta_{Y}\left(x_{1}, x_{2}\right)= \begin{cases}\theta_{Y 1} & \text { if }|x|<0.2 \\ \theta_{Y 1} & \text { if } 0.2<|x|<0.8,0 \leq N \phi-2\left[\frac{N \phi}{2 \pi}\right] \pi<\frac{2 \pi}{3} \\ \theta_{Y 2} & \text { if } 0.2<|x|<0.8, \quad \frac{2 \pi}{3} \leq N \phi-2\left[\frac{N \phi}{2 \pi}\right] \pi<2 \pi \\ \theta_{Y 1} & \text { if }|x|>0.8\end{cases}
$$

Here $N$ is the number of period of the patterns and $\phi$ is the angle from the $x$ axis to vector $(x, y)$. In Fig. 6, we show a droplet on such a chemically patterned surface.

In this example, we are interested in the macroscopic contact angle $\theta_{a}$ of a droplet on the surface. The most well-known equation to determine the apparent contact angle on chemically patterned surface is the Cassie's equation [9]. It reads

$$
\cos \theta_{a}=\lambda \cos \theta_{Y 1}+(1-\lambda) \cos \theta_{Y 2},
$$


Table 4: Verification of the modified Cassie equation.

\begin{tabular}{||c|c|c|c|c||}
\hline$\theta_{Y 1}$ & $\theta_{Y 2}$ & $\begin{array}{c}\text { Numerical } \\
\text { results }\end{array}$ & $\begin{array}{c}\text { Modified Cassie } \\
\text { equation }\end{array}$ & $\begin{array}{c}\text { Classic Cassie } \\
\text { equation }\end{array}$ \\
\hline $60^{\circ}$ & $120^{\circ}$ & $96.3^{\circ}$ & $99.6^{\circ}$ & $79.3^{\circ}$ \\
\hline $90^{\circ}$ & $120^{\circ}$ & $106.3^{\circ}$ & $109.5^{\circ}$ & $99.0^{\circ}$ \\
\hline $30^{\circ}$ & $60^{\circ}$ & $47.4^{\circ}$ & $51.5^{\circ}$ & $41.3^{\circ}$ \\
\hline
\end{tabular}

where $\lambda$ is the area fraction of material 1 in the surface. The equation is proved to be correct when one considers the global minimizer of the system. However, the equation is not correct when local minimizers are considered [2]. It is proved that the local minimizers can be described by a modified Cassie equation [49]. A similar equation has also been proposed in [33]. The modified Cassie equation reads

$$
\cos \theta_{a}=\tilde{\lambda} \cos \theta_{Y 1}+(1-\tilde{\lambda}) \cos \theta_{Y 2}
$$

where $\tilde{\lambda}$ is the line fraction of the projection of the contact line in material 1 . The definition is as follows. Suppose the homogenized contact line is a circle, then $\tilde{\lambda}$ is equal to the fraction of the length of the arc located in material 1 . Here we will verify the equation by numerical simulations.

We first consider the case $\theta_{Y 1}=\frac{\pi}{3}$ and $\theta_{Y 2}=\frac{2 \pi}{3}$. We test for different $N=15,20,30$. The initial shape of the droplet is a hemisphere with radius $r_{0}=0.5$. A typical numerical result is given in Fig. 6 for $N=20$. We could see that there are small oscillations near the contact line in the surface. The apparent contact angle is computed approximately as follows. Firstly, we search for a spherical surface which has minimal distance from the liquid surface. The apparent contact angle is the angle between the spherical surface and the bottom surface. In this way, we find that the apparent contact angles are respectively $94.9^{\circ}, 96.3^{\circ}$ and $96.3^{\circ}$ for $N=15,20,30$ cases. It is close to the value predicted by the modified Cassie equation that $\theta_{a}=\arccos (\cos (\pi / 3) / 3+2 \cos (2 \pi / 3) / 3) \approx 99.6^{\circ}$. The discrepancy between the theoretical and numerical results are due to numerical errors in the computation. In the calculations, we choose $h=\delta t=1 / 256$. In comparison, the contact angle computed by the Cassie equation is $\theta_{a}=\arccos (\lambda \cos (\pi / 3)+(1-\lambda) \cos (2 \pi / 3)) \approx 79.3^{\circ}$ with the area fraction of material 1 be $\lambda \approx 0.6858$.

We also make simulations for some other cases. The results are illustrated in Table 4 . We could find that the modified Cassie equation always fits much better with the numerical results than the classical Cassie equation.

\subsection{Wetting on geometrically rough surfaces}

We consider wetting on a geometrically rough solid surface. We consider several different shapes of the solid surface. The first surface is given by a function $z=h(x, y)$ 


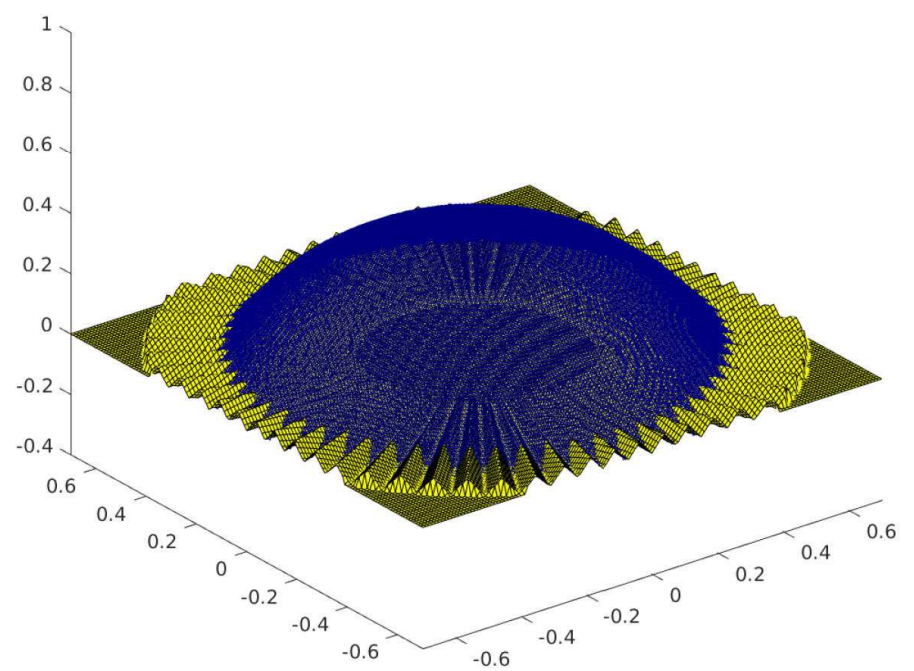

Figure 7: The shape of the droplet on a geometrically rough surface.

$$
h(x, y)= \begin{cases}0 & \text { if }|x|<0.3 \text { or }|x|>0.8 \\ \phi-2\left[\frac{N \phi}{2 \pi}\right] \pi / N & \text { if } 0.2<|x|<0.8,0 \leq N \phi-2\left[\frac{N \phi}{2 \pi}\right] \pi<\pi ; \\ 2\left(\left[\frac{N \phi}{2 \pi}\right]+1\right) \pi / N-\phi & \text { if } 0.2<|x|<0.8, \pi \leq N \phi-2\left[\frac{N \phi}{2 \pi}\right] \pi<2 \pi .\end{cases}
$$

The shape is carefully chosen so that it is radially symmetric as shown in Fig. 7. The slope of the rough surface is about 1 . We would like to verify the modified Wenzel equation [45]. If we assume that the contact line is also radially symmetric, the modified Wenzel's equation will be reduced to

$$
\cos \theta_{a}=R \cos \theta_{Y}
$$

where $R \approx \sqrt{2}$ is the ratio between the length of the contact line to its projection on the effective flat surface. We choose $\theta_{Y}=70^{\circ}$ so that the condition

$$
\left|\cos \theta_{Y}\right| \max \sqrt{1+|\nabla h|^{2}}<1
$$

is satisfied. The condition makes sure the Cassie-Baxter state does not appear. By the modified Wenzel's equation (4.5), we have $\theta_{a} \approx 61^{\circ}$. A typical numerical result is shown in Fig. 7. We could find that there are some oscillations of the contact line. We then compute an apparent contact angle by the same method as that in the case with a chemically patterned surface. The computed apparent contact angle is about $63.8^{\circ}$. We find that it is close to the theoretic prediction.

In the following, we will show some numerical results on a more general rough surface. The solid surface is given by a function $z=h(x, y)$ that

$$
h(x, y)=0.1 \sin (2 \pi N x) \sin (2 \pi N y),
$$



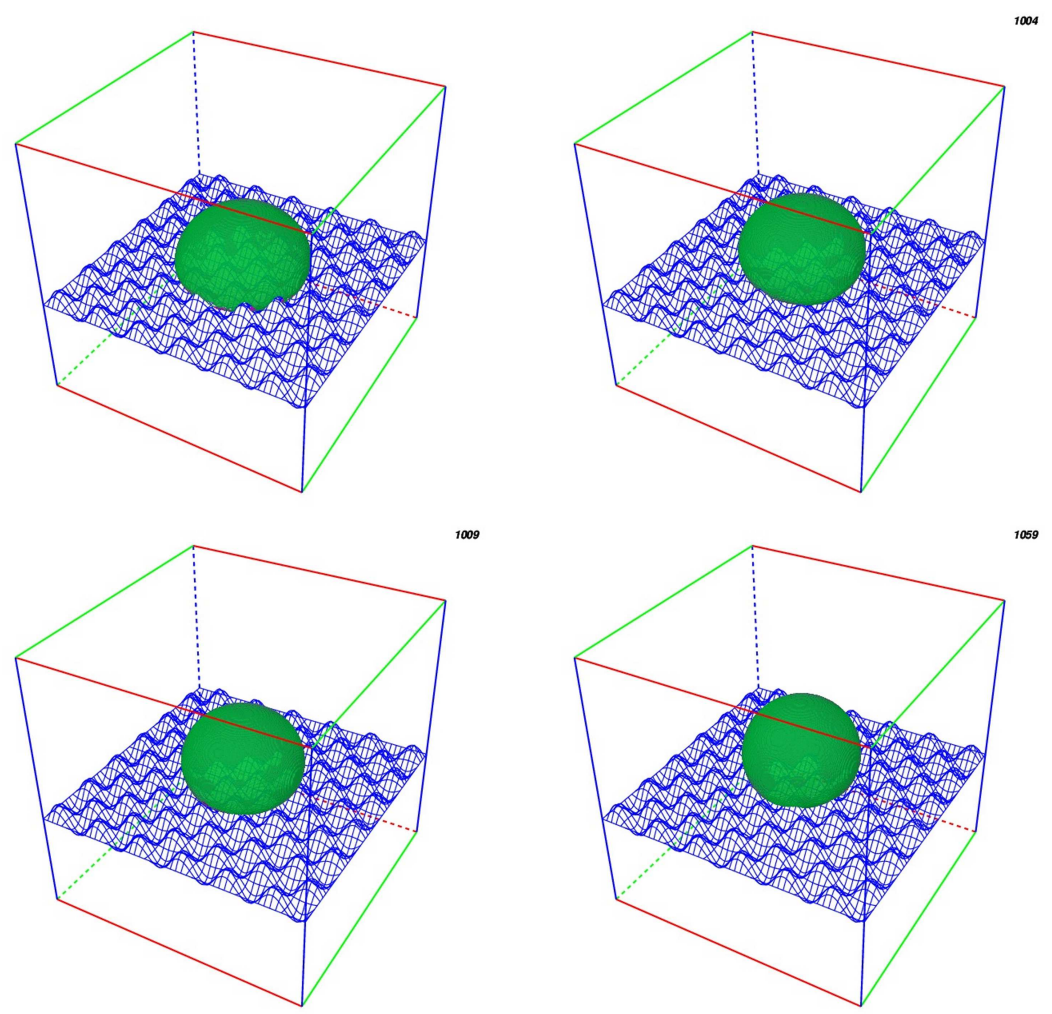

Figure 8: The shapes of a droplet on a general rough surface(from an initial state to an equilibrium state.

with $N=6$ and the Young's angle is chosen as $\theta_{Y}=135^{\circ}$. In Fig. 8, we show some numerical results. The initial shape of the droplet is almost a hemisphere. There is no air between the droplet and the solid surface. We could see that the droplet gradually gets to an equilibrium state, where the apparent contact angle is much larger than the Young's angle. This is related to the lotus effect. We could also see that there is some air trapped below the droplet. This is the so-called Cassie-Baxter state [9]. Our numerical method can naturally deal with the topology change in the transition from the Wenzel to CassieBaxter state.

\subsection{Droplet on curved solid surface}

In the last subsection, we will consider examples of some wetting problems on curved solid surfaces. We first consider wetting on the surface of a fiber. The solid surface is the surface of a thin cylinder. We assume that the surface is chemically inhomogeneous in the sense that the Young's angle has a constant gradient along the fiber surface. The droplet on such a surface will move due to the wetting gradient [5]. We will simulate this phenomena by our method. 

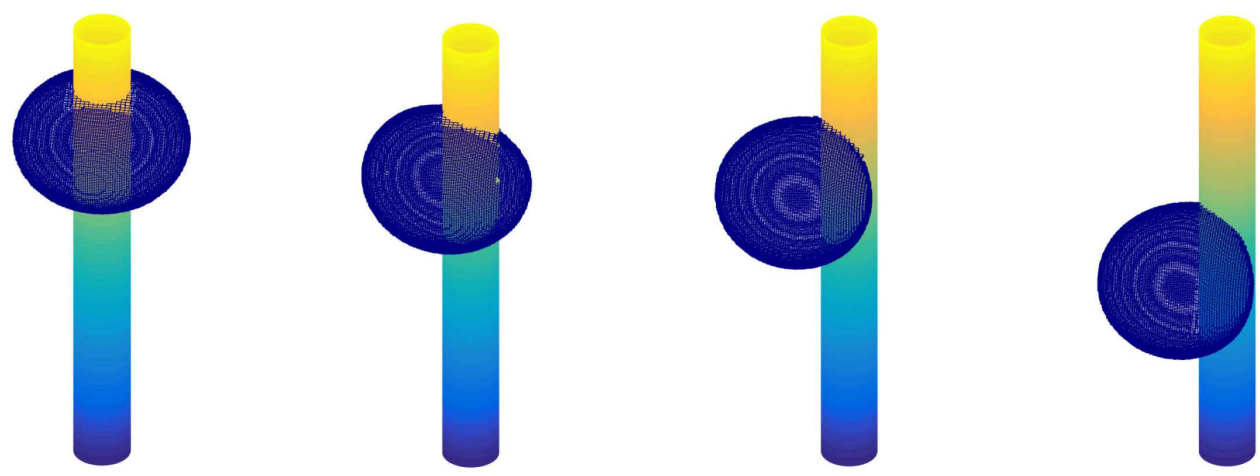

Figure 9: The shape of the droplet on a rod in different steps.

The set-up of the example is as follows. The radius of the fiber is 0.1. The length of the fiber is 2. Along the fiber, we set a coordinate system with the $z$ axis along the central line of the cylinder. The origin is in the middle of the central line so that $z \in(-1,1)$. The Young's angle on the surface is a function of $z$ given by

$$
\theta_{Y}(z)=\frac{(1+z)}{2} \frac{\pi}{3}+\frac{(1-z)}{2} \frac{2 \pi}{3} .
$$

The initial droplet is spherical as shown in the first sub-figure of Fig. 9. The spherical surface has a radius 0.3 and is centered at $(0,0,0.5)$. By the threshold dynamics method, the droplet changes positions and shapes in the direction of decreasing the total surface energy. Numerical results are shown in Fig. 9. We can see that the topology of the liquid surface changes in the process.

We then study motion of a droplet on a geometrically inhomogeneous surface, whose mean curvature is not constant [5]. As shown in Fig. 10, we consider wetting on the surface of a cone. The Young's angle is $\frac{\pi}{2}$. We put a small droplet in the outer surface of the cone. Evolution of the droplet is given in Fig. 10. We could see that when the droplet is put outside of the cone, the droplet will move outward. This is consistent with the previous physical experiments and analysis [24,28].

Finally, we would like to remark that the pictures in this subsection show qualitative properties of the motion of a droplet. We have not considered viscous dissipations in the fluid yet. For quantitative study on wetting problems, we may use a recently developed approximation method by the Onsager principle [21,46]. This will be left for future work.

\section{Conclusion}

In this paper, we propose an adaptive threshold dynamics method for wetting problems on complicated solid surfaces. The method is efficient and easy to implement. In every 

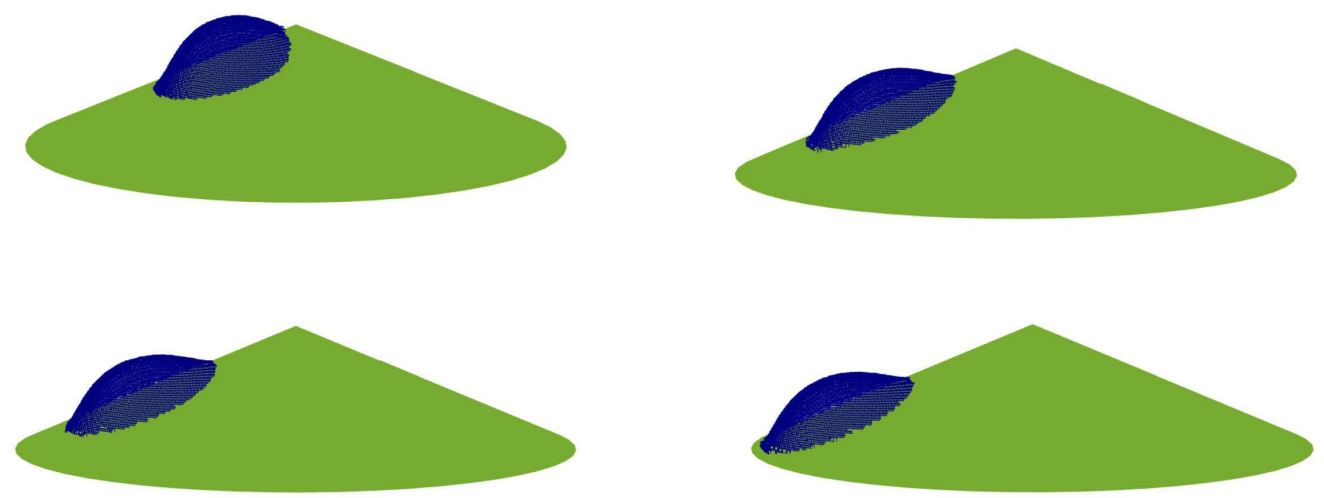

Figure 10: The shape of the droplet on the surface of a cone in different steps.

step, we only need to solve a linear heat equation up to a small time $\delta t$. The heat equation is discretized efficiently by an adaptive cell-centered finite volume method and the discrete equations can be solved with a fast multilevel composite grid iterative method. It is remarkable that, in contrast with a vertex-centered finite volume or finite element method, the cell-centered finite volume discretization is especially convenient for computing cell volumes and sorting cells by volumes. A time-adaptive technique is further used to improve the accuracy. The above numerical techniques might also be useful in other applications, like the multi-phase mean curvature flows in three dimensions.

Some numerical examples are presented to show the efficiency of the numerical method. We verify some previous theoretical analysis results for the apparent contact angles on rough or chemically inhomogeneous surfaces, which have not been fully understood in wetting $[14,16]$. Some interesting droplet motion problems on curved solid surfaces are studied qualitatively. The method can easily deal with topology changes which appear when the solid surface is complicated, e.g. in the case of the Wenzel-Cassie transition.

Finally, to further improve the accuracy of the method, one can use a signed distance function as that in [18]. The usage of the level-set function instead of the characteristic function may improve the convergence order far from the contact lines.

\section{Acknowledgments}

The authors would like to thank the anonymous reviewers for valuable suggestions that help us to revise the paper. The work was supported in part by NSFC grants DMS11971469, DMS-11771290, and the National Key R\&D Program of China under Grant 2018YFB0704304 and Grant 2018YFB0704300. 


\section{References}

[1] G. Alberti and G. Bellettini. A non-local anisotropic model for phase transitions: asymptotic behaviour of rescaled energies. Euro. J. Appl. Math., 9(03):261-284, 1998.

[2] G. Alberti and A. DeSimone. Wetting of rough surfaces: A homogenization approach. Proc. R. Soc. A, 451:79-97, 2005.

[3] D. Bonn, J. Eggers, J. Indekeu, J. Meunier, and E. Rolley. Wetting and spreading. Rev. Mod. Phys., 81:739-805, 2009.

[4] E. Bormashenko. A variational approach to wetting of composite surfaces: Is wetting of composite surfaces a one-dimensional or two-dimensional phenomenon? Langmuir, 25:10451-10454, 2009.

[5] E. Bormashenko. Wetting of flat gradient surfaces. J. colloid interface sci., 515:264-267, 2018.

[6] K. A. Brakke. The surface evolver. Exp. math., 1(2):141-165, 1992.

[7] J. H. Bramble, R. E. Ewing, J. E. Pasciak, and A. H. Schatz. A preconditioning technique for the efficient solution of problems with local grid refinement. Comput. Meth. Appl. Mech. Engrg., 67:149-159, 1988.

[8] W. L. Briggs, V. E. Henson, and S. F. McCormick. A Multigrid Tutorial (second edition). SIAM, 2000.

[9] A. Cassie and S. Baxter. Wettability of porous surfaces. Trans. Faraday Soc., 40:546-551, 1944.

[10] X. Chen, X.-P. Wang, and X. Xu. Analysis of the Cahn-Hilliard equation with relaxation boundary condition modelling contact angle. Arch. Rational Mech. Anal., 213:1-24, 2014.

[11] W. Choi, A. Tuteja, J. M. Mabry, R. E. Cohen, and G. H. McKinley. A modified CassieBaxter relationship to explain contact angle hysteresis and anisotropy on non-wetting textured surfaces. J. Colloid Interface Sci., 339:208-216, 2009.

[12] P.G. de Gennes. Wetting: Statics and dynamics. Rev. Mod. Phys., 57:827-863, 1985.

[13] K. Deckelnick, G. Dziuk, and C. M. Elliott. Computation of geometric partial differential equations and mean curvature flow. Acta numerica, 14:139-232, 2005.

[14] D. W. Drelich, L. Boinovich, E. Chibowski, C. D. Volpe, L. Hołysz, A. Marmur, and S. Siboni. Contact angles: History of over 200 years of open questions. Surface Innov., pages 1-25, 2019.

[15] M. Elsey and S. Esedoglu. Threshold dynamics for anisotropic surface energies. Math. Comp., 87(312):1721-1756, 2018.

[16] H. Y. Erbil. The debate on the dependence of apparent contact angles on drop contact area or three-phase contact line: a review. Surf. Sci. Rep., 69(4):325-365, 2014.

[17] S. Esedoglu and F. Otto. Threshold dynamics for networks with arbitrary surface tensions. Comm. Pure Appl. Math., 2015.

[18] S. Esedoglu, S. Ruuth, and R. Tsai. Diffusion generated motion using signed distance functions. J. Comput. Phys., 229(4):1017-1042, 2010.

[19] S. Esedoglu and R. Tsai. Threshold dynamics for the piecewise constant Mumford-shah functional. J. Comput. Phys., 211(1):367-384, 2006.

[20] L. C. Evans. Convergence of an algorithm for mean curvature motion. Indiana Math. J., 42(2):533-557, 1993.

[21] S. Guo, X. Xu, T. Qian, Y. Di, M. Doi, and P. Tong. Onset of thin film meniscus along a fibre. J. Fluid Mech., 865:650-680, 2019.

[22] K. Ishii. Optimal rate of convergence of the Bence-Merriman-Osher algorithm for motion by mean curvature. SIAM J. Math. Anal., 37(3):841-866, 2005.

[23] S. Jiang, D. Wang, and X. P. Wang. An efficient boundary integral scheme for the MBO threshold dynamics method via the NUFFT. J. Sci. Comput., 74:474-490, 2018. 
[24] W. Jiang, Y. Wang, D. J. Srolovitz, and W. Bao. Solid-state dewetting on curved substrates. Phy. Rev. Materials, 2(11):113401, 2018.

[25] C. Kublik, S. Esedoglu, and J. A. Fessler. Algorithms for area preserving flows. SIAM J. Sci. Comput., 33(5):2382-2401, 2011.

[26] S. T. Larsen and R. Taboryski. A cassie-like law using triple phase boundary line fractions for faceted droplets on chemically heterogeneous surfaces. Langmuir, 25(3):1282-1284, 2009.

[27] T. Laux and F. Otto. Convergence of the thresholding scheme for multi-phase meancurvature flow. Calc. Var. Partial Diff. Equ., 55(5):129, 2016.

[28] C. Lv, C. Chen, Y. C. Chuang, F. G. Tseng, Y. Yin, F. Grey, and Q. Zheng. Substrate curvature gradient drives rapid droplet motion. Phy. Rev. Lett.Cal. Var. Partial Diff. Equ., 113:026101, 2014.

[29] S. McCormick and J. Thomas. The fast adaptive composite grid (FAC) method for elliptic equations. Math. Comp., 46(174):439-456, 1986.

[30] B. Merriman, J. K. Bence, and S. J. Osher. Motion of multiple junctions: A level set approach. J. Comput. Phys., 112(2):334-363, 1994.

[31] B. Osting and D. Wang. A diffusion generated method for orthogonal matrix-valued fields. Math. Comp., 2019, DOI:https://doi.org/10.1090/mcom/3473.

[32] T. Qian, X.-P. Wang, and P. Sheng. Molecular scale contact line hydrodynamics of immiscible flows. Phy. Rev. E, 68(1):016306-15, July 2003.

[33] R. Raj, R. Enright, Y. Zhu, S. Adera, and E. N. Wang. Unified model for contact angle hysteresis on heterogeneous and superhydrophobic surfaces. Langmuir, 28:15777-15788, 2012.

[34] W. Ren. Wetting transition on patterned surfaces: transition states and energy barriers. Langmuir, 30:2879-2885, 2014.

[35] S. J. Ruuth. A diffusion-generated approach to multiphase motion. J. Comput. Phys., 145(1):166-192, 1998.

[36] S. J Ruuth and B. T. Wetton. A simple scheme for volume-preserving motion by mean curvature. J. Sci. Comput., 19(1-3):373-384, 2003.

[37] Steven J Ruuth and Barry Merriman. Convolution-thresholding methods for interface motion. J. Computat. Phys., 169(2):678-707, 2001.

[38] K. Svadlenka, E. Ginder, and S. Omata. A variational method for multiphase volumepreserving interface motions. J. Comput. Appl. Math., 257:157-179, 2014.

[39] D. Swartz and N. Yip. Convergence of diffusion generated motion to motion by mean curvature. Comm. Partial Differ. Equ., 42(10):1598-1643, 2017.

[40] D. Wang, H. Li, X. Wei, and X.-P. Wang. An efficient iterative thresholding method for image segmentation. J. Comput. Phys., 350:657-667, 2017.

[41] D. Wang, X. Xu, and X.-P. Wang. An improved threshold dynamics method for wetting dynamics. J. Comput. Phys., 392:291-310, 2019.

[42] Dong Wang, Shidong Jiang, and Xiao-Ping Wang. An efficient boundary integral scheme for the threshold dynamics method ii: Applications to wetting dynamics. J. Sci. Comp., 81(3):1860-1881, 2019.

[43] R. N. Wenzel. Resistance of solid surfaces to wetting by water. Ind. Eng. Chem., 28:988-994, 1936.

[44] D. E. Womble. A front-tracking method for multiphase free boundary problems. SIAM J. Numer. Anal., 26(2):380-396, 1989.

[45] X. Xu. Modified Wenzel and Cassie equation for wetting on rough surfaces. SIAM J. Appl. Math., 76:2353-2374, 2016.

[46] X. Xu, Y. Di, and M. Doi. Variational method for liquids moving on a substrate. Phy. Fluids, 
28(8):087101, 2016

[47] X. Xu, D. Wang, and X.-P. Wang. An efficient threshold dynamics method for wetting on rough surfaces. J. Comput. Phys., 330:510-528, 2017.

[48] X. Xu and X. P. Wang. Analysis of wetting and contact angle hysteresis on chemically patterned surfaces. SIAM J. Appl. Math., 71:1753-1779, 2011.

[49] X. Xu and X. P. Wang. The modified Cassie's equation and contact angle hysteresis. Colloid Polym. Sci., 291:299-306, 2013.

[50] T. Young. An essay on the cohesion of fluids. Philos. Trans. R. Soc. London, 95:65-87, 1805.

[51] H. Zhao, T. Chan, B. Merriman, and S. J. Osher. A variational level set approach to multiphase motion. J. Comput. Phys., 127(1):179-195, 1996. 